\title{
Índice de Qualidade da Dieta: avaliação da adaptação e aplicabilidade
}

\section{Healthy Eating Index: evaluation of adapted version and its applicability}

\author{
Regina Mara FISBERG ${ }^{1}$ \\ Betzabeth SLATER ${ }^{1}$ \\ Rodrigo Ribeiro BARROS 1 \\ Fernão Dias de LIMA ${ }^{1}$ \\ Chester Luiz Galvão CESAR ${ }^{1}$ \\ Luana CARANDINA² \\ Marilisa Berti de Azevedo BARROS 3 \\ Moisés GOLDBAUM ${ }^{4}$
}

\section{RE S U M O}

\section{Objetivo}

O objetivo deste estudo foi adaptar e aplicar o Healthy Eating Index norte-americano para avaliar a qualidade da dieta de indivíduos ( $n=50)$ moradores em Botucatu, São Paulo, Brasil.

\section{Método}

O consumo alimentar foi medido por meio do método Recordatório 24 horas e, para avaliação, foi utilizado o Índice de Qualidade da Dieta adaptado. O índice foi obtido por uma pontuação distribuída em dez componentes que caracterizam diferentes aspectos de uma dieta saudável.

\section{Resultados}

O valor médio do Índice de Qualidade da Dieta foi de 51,5, com 12\% dos indivíduos apresentando dieta "saudável"; 74\%, em dietas "necessitando modificações" e 14\%, em dieta "inadequada". A análise do

\footnotetext{
1 Departamentos de Nutrição, de Epidemiologia e de Estatística, Faculdade de Saúde Pública, Universidade de São Paulo. Av. Dr. Arnaldo, 715, Cerqueira César, 01246-904, São Paulo, SP, Brasil. Correspondência para/Correspondence to: R.M. FISBERG. E-mail: rfisberg@usp.br

2 Departamento de Saúde Pública, Faculdade de Medicina, Universidade Estadual Paulista. Botucatu, SP, Brasil.

${ }^{3}$ Departamento de Medicina Preventiva e Social, Faculdade de Ciências Médicas, Universidade Estadual de Campinas. Campinas, SP, Brasil.

${ }^{4}$ Departamento Medicina Preventiva, Faculdade de Medicina, Universidade de São Paulo. São Paulo, SP, Brasil. Apoio: FAPESP, processo: 98/14099-7.
} 
coeficiente de correlação entre os escores do Índice de Qualidade da Dieta apresentou associação inversa estatisticamente significante $(p<0,05)$ com a porcentagem de gordura total, a porcentagem de gordura saturada, o colesterol e o sódio. Apresentaram associação positiva estatisticamente significante em relação ao Índice de Qualidade da Dieta o retinol e a fibra.

\section{Conclusão}

Os resultados indicam a viabilidade de aplicação do Índice de Qualidade da Dieta na população estudada.

Termos de indexação: qualidade da dieta, índice de qualidade da dieta, dieta, nutrição.

\section{A B S T R A C T}

\section{Objective}

This study was to adapt and to applied the U.S.A.'s Healthy Eating Index to evaluate the diet quality of individuals ( $n=50$ ) residing in Botucatu, São Paulo, Brazil.

\section{Methods}

The food intake was measured by the 24-hour dietary recall method, and evaluated by an adapted Healthy Eating Index. The index was obtained by a score of the distribution of the ten components of the considered healthy diet.

\section{Results}

The mean Healthy Eating Index was 51,5; it was found that 12 percent of individuals had "good" diets; 74 percent, had diets that "needed improvement"; and 14 percent, had "poor" diets. There were a negative and significant correlation $(p<0,05)$ between the Healthy Eating Index and the total dietary fat, fatty acids, cholesterol and sodium. The mean retinol and fiber intakes, presented a positive and significant correlation with the Healthy Eating Index.

\section{Conclusion}

The results showed that the use of Healthy Eating Index in a target population is feasible.

Index terms: quality of diet, healthy eating index, diet, nutrition.

\section{N T R O D U Ç Ã O}

É amplamente aceito que a dieta pode influenciar o estado de saúde do indivíduo. Estudos observacionais têm evidenciado o papel da dieta no desenvolvimento de doenças cardiovasculares, diabetes tipo II e câncer ${ }^{1,2}$; por outro lado, uma dieta variada, pobre em gorduras e rica em vitamina $C$, fibras e $\beta$-caroteno, está associada ao menor risco de desenvolvimento de alguns tipos de câncer ${ }^{3,4}$.

Paralelamente, tem-se procurado desenvolver ferramentas úteis e válidas que ao mesmo tempo permitam relacionar o consumo dietético com à incidência das doenças crônicas não transmissíveis e aos eventos de morbidade e mortalidade. Muitos dos instrumentos que a literatura científica vem publicando, focalizam o consumo de nutrientes de forma específica e isolada, como tem sido o caso da gordura dietética e outros componentes da dieta' ${ }^{1}$.

Mertz ${ }^{5}$ afirma que a abordagem científica que avalia a quantidade dos nutrientes e seus efeitos na saúde dos indivíduos, geralmente apresenta a desvantagem de analisar a dieta separadamente do contexto sociocultural. 
Em muitas sociedades de baixa renda observa-se um padrão dietético com alto conteúdo de gorduras totais, colesterol e açúcar refinado e com baixo teor de fibra alimentar. Este padrão, denominado de "dieta ocidental", está estreitamente relacionado a uma vida sedentária e à ocorrência de doenças crônicas não transmissíveis (DCNT). Países como o Brasil e a China ilustram situações em que, dentro de uma mesma população, padrões nutricionais de excesso coexistem com padrões de déficit - fome e desnutrição $0^{2,6}$.

Os dados de consumo alimentar são coletados em diferentes níveis (nacional, familiar ou individual) e por múltiplas razões, que vão desde o conhecimento da etiologia da doença até o monitoramento da saúde nutricional da população.

Informações sobre o consumo alimentar da população são escassas no Brasil, que, ainda hoje, conta apenas com os dados provenientes do Estudo Nacional sobre Despesa Familiar (ENDEF) realizado em uma amostra probabilística de 55 mil domicílios em todo o país. Estudos mais recentes, de cunho econômico, as Pesquisas de Orçamento Familiar (POFs) constituem uma fonte importante de dados sobre o consumo alimentar, obtidos a partir da focalização sobre a despesa gerada pela compra de alimentos. Conta-se também com o Estudo Multicêntrico sobre Consumo Alimentar realizado pelo Instituto Nacional de Alimentação e Nutrição e Ministério da Saúde (INAN-MS) e coordenado pelo Núcleo de Estudos e Pesquisas em Alimentação (NEPA) no ano de 1996, em cinco cidades do país. Este estudo informa sobre o consumo alimentar familiar e teve como objetivo medir entre as famílias a disponibilidade de cem gêneros alimentícios ${ }^{7}$.

Como se pode observar, as pesquisas apresentam certas vantagens e outras limitações. A principal vantagem é que inquéritos dietéticos, como a pesagem direta de alimentos utilizada pela ENDEF, proporcionam uma medida direta e precisa do consumo alimentar em nível individual; se realizados com regularidade, proporcionam ainda informações sobre séries temporais, essenciais para identificar mudanças no padrão alimentar, tanto por estratos socioeconômicos, como por regiões geográficas - informações úteis para posterior formulação de políticas nutricionais em saúde pública ${ }^{8}$. Em contrapartida, a limitação destes estudos está em não avaliar a dieta habitual, tornando-se difícil relacionar-se a dieta com a saúde nutricional da população.

O Índice de Qualidade da Dieta (Healthy Eating Index) proposto por Kennedy et al. ${ }^{9}$ foi considerado pela American Dietetic Association, um instrumento adequado para medir a qualidade global da alimentação na população. A partir de então, profissionais da área da nutrição e saúde passaram a contar com um índice válido em que se basear para projetar atividades que promovam hábitos alimentares saudáveis ${ }^{10}$.

Considerando a importância da realização de estudos de consumo alimentar populacionais e a necessidade de que estes forneçam informações úteis para construir indicadores da saúde nutricional da população, este estudo teve como objetivo principal avaliar a adaptação e aplicabilidade do Healthy Eating Index, proposto por Kennedy et al. ${ }^{9}$, como medida da qualidade da dieta de um grupo de moradores da cidade de Botucatu, SP.

\section{CASUÍSTICA E MÉTODOS}

Trata-se de um estudo de caráter metodológico que foi conduzido em 50 participantes, indivíduos de ambos os sexos, de todas as faixas etárias com exclusão apenas dos menores de 1 ano. O estudo piloto que foi desenvolvido na cidade de Botucatu, SP, em novembro/dezembro de 2000, como parte integrante do Inquérito de Saúde do Estado de São Paulo (ISA-SP) realizado em municípios do Estado de São Paulo. Os entrevistados foram assim distribuídos: 8 crianças de 1 a 11 anos, de ambos os sexos; 7 adolescentes de 12 a 19 anos, do sexo masculino; 7 adolescentes de 12 a 19 anos, do sexo feminino; 7 indivíduos 
de 20 a 59 anos, do sexo masculino; 7 indivíduos de 20 a 59 anos, do sexo feminino; 8 homens de 60 anos e mais; 8 mulheres de 60 anos e mais.

Realizaram-se visitas domiciliares, pelas quais se obteve dados sobre o consumo alimentar dos indivíduos selecionados. As entrevistas foram pré-agendadas e transcorreram em novembro/ dezembro de 2000.

Realizou-se inquérito alimentar utilizando o método Recordatório 24 horas (R24h); no caso das crianças, o inquérito foi respondido pela mãe ou pessoa responsável pela alimentação. Utilizou-se o método passo a passo, adaptado de Thompson \& Byers ${ }^{11}$. Os entrevistadores foram treinados para a padronização na coleta de dados e na utilização do formulário padrão para aplicação do R24h, recebendo ainda um manual explicativo.

Calculou-se a composição química dos alimentos componentes dos recordatórios de cada indivíduo, utilizando o Virtual Nutri. Posteriormente, agrupando-se os alimentos de acordo com sua composição, estabeleceu-se uma relação entre os resultados obtidos sobre o consumo diário e os grupos de alimentos constantes da Pirâmide Alimentar, adaptada de Philippi et al. ${ }^{12}$. Preparações que envolvem mais de um grupo de alimentos, como sanduíches, pizzas, massas recheadas e sucos enriquecidos, foram desmembradas e seus ingredientes, classificados em cada grupo correspondente.

Para avaliação do consumo alimentar foi utilizado o Índice de Qualidade da Dieta (IQD) adaptado de Kennedy et al. ${ }^{9}$. O índice foi obtido por uma pontuação distribuída entre os dez componentes que caracterizam diferentes aspectos de uma dieta saudável. As mudanças realizadas para adaptação do índice foram: a utilização do guia alimentar proposto por Philippi et al..$^{12}$ como parâmetro para os componentes de 1 a 5; e o componente 10 como variedade da dieta elaborada a partir dos dados obtidos neste estudo. Todos os componentes foram avaliados e pontuados de zero a dez, sendo que os valores intermediários foram calculados proporcionalmente ao consumido. A qualidade da dieta foi determinada segundo três categorias definidas pela distribuição dos escores encontrados na população-alvo.

- Componentes 1-5: Grupos de Alimentos (Cereais, pães, tubérculos e raízes; verduras e legumes; frutas; leite e produtos lácteos; carnes, ovos e feijão). Mede o grau de adequação do consumo de cada um dos cinco grupos de alimentos, estabelecidos pelo guia alimentar, segundo faixa etária. Consumido o mínimo recomendado pelo guia, o indivíduo recebe 10 pontos; e quando não atender às recomendações, zero. O consumo de um número intermediário de porções (entre o não consumo e o mínimo recomendado) foi pontuado proporcionalmente.

- Componente 6: Gordura Total. O valor mínimo (zero) corresponde à ingestão de lipídeos totais em quantidade igual ou superior a $45 \%$ do Valor Calórico Total da dieta (VCT), enquanto que 10 é atribuído ao consumo de $30 \%$ ou menos do VCT, uma vez que a faixa compreendida entre $30 \%$ e $45 \%$ seria aceitável, segundo as recomendações do Dietary Guidelines for Americans $^{13}$.

- Componente 7: Gordura Saturada. A pontuação, assim como com os lipídeos totais, é dada conforme a importância da porcentagem da gordura saturada no VCT. Atribui-se o valor mínimo quando o indivíduo ingere $15 \%$ ou mais do VCT em gordura saturada; atribuem-se 10 pontos a uma dieta de $10 \%$ ou menos do VCT referentes às mesmas recomendações do Dietary Guidelines for Americans.

- Componente 8: Colesterol. Segundo o Committee on Diet and Health ${ }^{14}$, a quantidade recomendada de colesterol na dieta seria de $300 \mathrm{mg} / \mathrm{dia}$ a $450 \mathrm{mg} / \mathrm{dia}$, sendo o primeiro valor equivalente à pontuação máxima, e o segundo, à pontuação mínima.

- Componente 9: Sódio. A ingestão de sódio foi pontuada de zero ( $4800 \mathrm{mg} / \mathrm{dia}$, ou mais) a dez (2400mg/dia, ou menos), baseado também nas recomendações do Committee on Diet and Health ${ }^{14}$. 
Componente 10: Variedade da Dieta. Foi medida levando-se em conta os diferentes tipos de alimentos consumidos durante um dia. A escala foi elaborada em função do consumo mínimo de 5 alimentos e um máximo de 15 alimentos diferentes por dia. Para o consumo inferior a 5 foi estabelecida pontuação zero e para consumo igual ou superior a 15, pontuação dez.

Depois de definidas as bases teóricas deste instrumento de pesquisa, foi desenvolvido em linguagem de SPSS (versão 8) para Windows, um programa de processamentos de dados "IQD-INDEX" que permite calcular e atribuir a pontuação para cada indivíduo.

O escore total dos indivíduos foi dividido em três categorias: abaixo ou igual a 40 pontos - dieta "inadequada"; entre 41 e 64 pontos - dieta que "necessita de modificação"; e igual ou superior a 65 pontos - dieta "saudável". Foram descritos também os valores médios obtidos de cada componente da dieta analisado e calculou-se o coeficiente de correlação de Pearson entre o IQD e as variáveis dietéticas (energia, \% de gordura total, \% de gordura saturada, colesterol, sódio, retinol, ferro e fibra).

\section{RES U LTA DOS}

A média dos escores atribuídos à população estudada foi de 51,5. Dos indivíduos avaliados, $12 \%$ apresentam dieta saudável, $74 \%$ seguem uma dieta que necessita de modificações e 14\%, dieta inadequada. Os valores de energia não diferiram entre as categorias de dieta saudável e inadequada. Entretanto, os valores observados para outros componentes, tais como porcentagem de gordura total, porcentagem de gordura saturada, colesterol e sódio, aumentaram à medida que os escores da dieta diminuíram, indicando dieta inadequada. Por outro lado, com o aumento dos escores da dieta, os valores de retinol, ferro e fibra também se elevaram (Tabelas 1a, 1b).

Os valores médios de pontos dos diferentes componentes do IQD apresentaram-se baixos para verduras e legumes e gordura saturada; valores intermediários, para leite e produtos lácteos, carnes, ovos e feijões; os mesmos valores médios apresentaram-se altos para cereais, frutas, colesterol, sódio e para a variedade de alimentos. Os maiores percentuais de observações com valor zero foram atribuídos ao componente frutas e à porcentagem de gordura saturada; entretanto, $60 \%$ dos indivíduos apresentaram pontuação máxima para colesterol na dieta (Tabela 2).

Tabela 1a. Consumo médio de energia, gordura total, gordura saturada e colesterol segundo categorias do Índice de Qualidade da Dieta de indivíduos moradores de Botucatu. 2001.

\begin{tabular}{lccccc}
\hline IQD Escore & $\mathrm{n}$ & Energia (kcal) & Gordura total (\%) & Gordura saturada (\%) & Colesterol (mg) \\
\hline Média 51,5 (11,8) & 50 & 2132,7 & 36,7 & 20,3 & 278,8 \\
Escore $\geq 65$ & 6 & 2911,0 & 28,2 & 16,7 & 264,7 \\
Escore 64 - 41 & 37 & 1847,1 & 35,5 & 20,5 & 255,1 \\
Escore $\leq 40$ & 7 & 2975,9 & 50,3 & 22,3 & 387,4 \\
\hline
\end{tabular}

Tabela 1b. Consumo médio de micronutrientes segundo categorias do Índice de Qualidade da Dieta de indivíduos moradores de Botucatu. 2001.

\begin{tabular}{lcccc}
\hline IQD Escore & Sódio $(\mathrm{mg})$ & Retinol $(\mathrm{mcg}$ ER) & Ferro $(\mathrm{mg})$ & Fibra $(\mathrm{g})$ \\
\hline Média $51,5(11,8)$ & 2852,1 & 781,8 & 11,5 & 10,7 \\
Escore $\geq 65$ & 3544,2 & 2133,3 & 18,7 & 17,8 \\
Escore $64-41$ & 2430,4 & 624,1 & 10,1 & 10,0 \\
Escore $\leq 40$ & 4487,7 & 457,4 & 12,6 & 8,1 \\
\hline
\end{tabular}


Tabela 2. Média e desvio-padrão dos escores dos componentes do Índice de Qualidade da Dieta de indivíduos moradores de Botucatu. 2001

\begin{tabular}{lcccc}
\hline Componentes & Média $(\mathrm{dp})$ Escore & \% de observações com escore 0 & \% de observações com escore 10 \\
\hline Cereais, pães e raízes & $7,0 \pm 2,5$ & 2 & 20 \\
Verduras e legumes & $1,9 \pm 2,3$ & 11 & 6 \\
Frutas & $8,0 \pm 1,9$ & 74 & 8 \\
Leites e produtos lácteos & $5,3 \pm 3,3$ & 36 & 42 \\
Carnes, ovos e feijões & $6,7 \pm 3,7$ & 2 & 28 \\
Gordura total & $5,4 \pm 4,7$ & 26 & 2 \\
Gordura saturada & $1,2 \pm 2,6$ & 78 & 60 \\
Colesterol & $7,6 \pm 3,5$ & 12 & 52 \\
Sódio & $7,5 \pm 3,5$ & 12 & 54 \\
Variedade de alimentos & $8,6 \pm 2,1$ & 2 & \\
\hline
\end{tabular}

Tabela 3. Coeficiente de correlação entre Índice de Qualidade da Dieta e energia e nutrientes da dieta de indivíduos moradores de Botucatu. 2001.

\begin{tabular}{lcc}
\hline Nutrientes & $r$ & $P$ \\
\hline Energia & $-0,11$ & 0,459 \\
Porcentagem de gordura total & $-0,70$ & 0,000 \\
Porcentagem de gordura saturada & $-0,29$ & 0,046 \\
Colesterol & $-0,33$ & 0,021 \\
Sódio & $-0,28$ & 0,049 \\
Retinol & 0,38 & 0,007 \\
Ferro & 0,21 & 0,138 \\
Fibra & 0,30 & 0,034 \\
\hline
\end{tabular}

A análise do coeficiente de correlação entre os escores do IQD demonstrou associação inversa estatisticamente significante $(p<0,05)$ com a porcentagem de gordura total, a porcentagem de gordura saturada, o colesterol e o sódio; não se observou associação significante com energia. Apresentaram associação positiva estatisticamente significante em relação ao IQD o retinol e a fibra, e não se observou significância para o ferro (Tabela 3).

Pode-se observar (Tabela 4) que os indivíduos avaliados apresentam dietas com alto

Tabela 4. Distribuição percentual dos indivíduos moradores de Botucatu segundo as recomendações dietéticas preconizadas. 2001.

\begin{tabular}{lcc}
\hline Recomendação** & Consumo & Percentual (\%) \\
\hline Reduzir o consumo de gordura total para menos de $30 \%$ da & $\leq 30 \%$ & 28 \\
energia & $30-45 \%$ & 50 \\
& $\geq 45 \%$ & 22 \\
Reduzir o consumo de Ácidos Graxos Saturados para menos de & $\leq 10 \%$ & 2 \\
$10 \%$ da energia & $10-15 \%$ & 22 \\
& $\geq 15 \%$ & 76 \\
Reduzir o consumo de colesterol para menos de 300mg por dia & $\leq 300 \mathrm{mg}$ & 36 \\
& $300-450 \mathrm{mg}$ & 14 \\
Limite de consumo diário de sódio para 2400mg ou menos & $\geq 400 \mathrm{mg}$ & 52 \\
& $2400-4500 \mathrm{mg}$ & 38 \\
Consumir 5 ou mais porções diárias de vegetais e frutas & $\geq 3400 \mathrm{mg}$ & 10 \\
\hline
\end{tabular}

\footnotetext{
${ }^{(* *)}$ National Research Council14.
} 
teor de gordura total e saturada, teor um pouco menos elevado de colesterol e sódio, além de baixo consumo de vegetais e frutas.

\section{I S C U S S Ã O}

O objetivo deste estudo foi o de adaptar e aplicar O IQD, com as modificações necessárias para nossa população, além de criar rotinas computadorizadas para viabilizar sua utilização. Embora os indivíduos tenham sido avaliados como um só grupo apesar das diferentes idades, sexos e estratos sociais, os resultados demonstraram aplicabilidade do IQD.

O IQD possibilita a observação da dieta de forma geral, analisando-se vários componentes e não simplesmente variáveis dietéticas específicas. O índice agrupa os indivíduos segundo as categorias de consumo alimentar permitindo possíveis associações com variáveis tais como: idade, renda e escolaridade, entre outras.

Alguns trabalhos na literatura apresentam a descrição de outros índices similares ao proposto por Bowman et al. ${ }^{10}$, na tentativa de determinar a ingestão alimentar de grupos de indivíduos, segundo uma medida-resumo, representativa de várias características da dieta, para monitorar os padrões alimentares.

Os indivíduos avaliados neste estudo consumiram quantidades semelhantes de calorias nas três categorias estabelecidas para o índice, porém apresentaram dietas qualitativamente opostas em relação aos nutrientes "saudáveis". Resultados similares foram descritos por Patterson et al. ${ }^{15}$ e Haines et al. ${ }^{16}$, que avaliaram a correlação da qualidade da dieta com outros índices propostos e demonstraram a relação entre o número de escores obtidos e o valor nutritivo da dieta.

Neste estudo, os valores médios do escore foram elevados para alguns componentes da dieta, como observado para frutas; porém, o percentual de indivíduos (26\%) que consumiram estes alimentos foi baixo, mostrando que alguns alimentos são consumidos por um reduzido número de indivíduos, porém em grandes quantidades.
Os valores percentuais de observações com escore zero foram superiores aos descritos por Kennedy et al. ${ }^{9}$ para todos os componentes do índice, exceção feita ao componente variedade. Ressalta-se que este estudo foi aplicado a um número pequeno de indivíduos; no entanto, a pontuação média para alguns componentes (vegetais e gordura saturada) e o percentual de indivíduos com escore zero, alertam para a existência de intensas distorções da dieta alimentar e revelam a importância da aplicação do índice em grupos maiores, com o intuito de analisar a qualidade da nutrição em subgrupos da população, visando à intervenção.

No estudo em questão o IQD teve alta correlação com a porcentagem de gordura na dieta; apresentou correlações mais baixas, porém estatisticamente significantes, com a quantidade de nutrientes ingerida, estabelecendo a relação entre o índice e seus componentes. Hann et al. ${ }^{17}$, estudando um grupo de mulheres, validaram o IQD em relação aos seus componentes e biomarcadores. Os valores altos de IQD foram associados à variedade da dieta, alta ingestão de frutas, baixa ingestão de gordura total e gordura saturada, e à alta concentração plasmática de $\alpha$-caroteno, $\beta$-caroteno e vitamina $C$, entre outros elementos.

Da população-alvo avaliada neste estudo, a maioria não segue as recomendações dietéticas preconizadas, o que pode redundar em problemas de saúde para os indivíduos em questão. Estudos realizados nos últimos anos já descreveram a importância de fatores de risco como hipertensão arterial, hábito de fumar, níveis de colesterol sérico, excesso de peso, sedentarismo, etilismo e dieta inadequada, na determinação das doenças crônicas não transmissíveis ${ }^{1,18,19}$.

O IQD constitui uma medida global da qualidade da alimentação, representando um instrumento com amplo potencial de uso na epidemiologia nutricional, útil para a descrição e o monitoramento do padrão alimentar da população, e para a avaliação das intervenções realizadas. 


\section{REFERÊ NCIAS}

1. Willet WC. Nutritional Epidemiology. 2nd ed. New York: Oxford University Press; 1998.

2. World Health Organization. Study Group on Diet, Nutrition and Prevention of Noncommunicable Diseases. Diet, nutrition and the prevention of chronic diseases: report of a WHO study group. Geneva: World Health Organization; 1990.

3. Burr ML. Antioxidants and cancer. J Hum Nutr Diet 1994; 7:409-16.

4. Flagg EW, Coates RJ, Greenberg RS. Epidemiologic studies of antioxidants and cancer in humans. J Am Coll Nutr 1995; 14:419-27.

5. Mertz W. Foods and nutrients. J Am Diet Assoc 1984; 84:769-70.

6. Popkin BM. Nutritional patterns and transitions. Popul Dev Rev 1993; 19:138-57.

7. Instituto Nacional de Alimentação e Nutrição. Estudo multicêntrico sobre consumo alimentar. Cad Deb 1997. Volume especial.

8. Mondini L, Monteiro CA. Mudanças no padrão de alimentação. In: Monteiro CA, organizador. Velhos e novos males da saúde no Brasil. São Paulo: Hucitec; 1995. p.79-89.

9. Kennedy ET, Ohls J, Carlson S, Fleming K. The Healthy Eating Index: design and applications. J Am Diet Assoc 1995; 95:1103-9.

10. Bowman AS, Lino M, Gerrior AS, Basiotis PP. The Healthy Eating Index: 1994-96. US Department of Agriculture, Center for Nutrition Policy and Promotion. CNPP-5. [online] 1998 [cited 2000 Dec 15]. Available from: http://www.USDA.gov/fcs/ cnpp.htm

11. Thompson FE, Byers T. Dietary assessment resource manual. J Nutr 1994; 124(Suppl):2245-317.
12. Philippi ST, Latterza AR, Cruz ATR, Ribeiro LC. Pirâmide alimentar adaptada: guia para escolha dos alimentos. Rev Nutr 1999; 12:65-80.

13. US Department of Agriculture, Agricultural Research Service. Report of the Dietary Guidelines Advisory Committee on the Dietary Guidelines for Americans. Washington, DC: U.S. Dept of Agriculture/Agricultural Research Service; 1995.

14. National Research Council. Committe On Diet And Health. Diet and health: implications for reducing chronic disease risk. Washington: National Academy Press; 1989.

15. Patterson RE, Haines PS, POPKIN BM. Diet Quality Index: capturing a multidimensional behavior. J Am Diet Assoc 1994; 94:57-64.

16. Haines PS, Siega-Riz AM, Popkin BM. The Diet Quality Index revised: a measurement instrument for populations. J Am Diet Assoc 1999; 99:697704.

17. Hann CS, Rock CL, King I, Drewnowski A. Validation of the Healthy Eating Index with use of plasma biomarkers in a clinical sample of women. Am J Clin Nutr 2001; 74:479-86.

18. Neumann AILCP. Consumo de alimentos de risco e proteção para doenças cardiovasculares entre funcionários públicos estaduais do município de São Paulo [dissertação]. São Paulo: Faculdade de Saúde Pública, Universidade de São Paulo; 2000.

19. Rego RA, et al. Fatores de risco para doenças crônicas não transmissíveis: inquérito domiciliar no Município de São Paulo, SP (Brasil): metodologia e resultados preliminares. Rev Saude Publica 1990; 24:277-85.

Recebido para publicação em 22 de outubro de 2002 e aceito em 27 de outubro de 2003. 